\title{
Clinical application of ultrasound-guided core needle biopsy with multiple punches in the diagnosis of lymphoma
}

\author{
Ying $\mathrm{He}^{1}$, Xiuzhen $\mathrm{Ji}^{1}$, Yanggui Xie ${ }^{2}$, Bosheng $\mathrm{He}^{3^{*}}$, Xiaohong Xu ${ }^{4}$, Xudong Chen ${ }^{5}$ and Qin Zhang ${ }^{1}$
}

\begin{abstract}
Background: The purpose of this study is to investigate the feasibility, accuracy, and limitations of ultrasound (US)-guided core needle biopsy (CNB) with multiple punches in the diagnosis of lymphoma in the whole body.

Methods: From March 2007 to October 2013, US-guided CNB with multiple punches was performed by well-experienced radiologists in 110 patients (CNB group), and surgical biopsy was carried out in 95 patients (surgical group). The differences of accuracy rate between the two groups in the diagnosis of lymphoma and its subtypes were examined with Fisher's exact test.

Results: There were no statistical differences between the CNB group and the surgical group in the diagnostic accuracy rate of lymphoma, as well as its subtypes in superficial and deep masses. In addition, in the CNB group, there were no statistical differences between different lengths of lesions in the diagnosis accuracy rate of lymphoma and its subtypes.

Conclusions: US-guided CNB with no less than three punches is an accurate, safe, minimally invasive, non-radiological, fast, and cost-effective method in the evaluation of lymphoma and its subtypes as compared with surgical approach. It should be considered as the acceptable alternative to surgical biopsy to obtain histopathological samples in the patients with suspected lymphoma.
\end{abstract}

Keywords: Lymphoma, Ultrasound-guided, Core needle biopsy, Multiple punches, Accuracy rate

\section{Background}

Different subtypes of lymphoma vary in clinical manifestations, treatment, and prognosis; therefore, early diagnosis and histological classification are crucial for the assignment of therapeutic schedule [1]. In the past, confirmed diagnosis and classification of lymphoma mainly relied on surgical biopsy which not only increased the patients' pains but also added up to their medical costs [2]. In recent years, minimally invasive techniques are gradually gaining recognition and have been widely used in the diagnoses of lymphoma.

Among the minimally invasive techniques, core needle biopsy (CNB) has been recognized as an alternative technique for diagnosing and subclassing the malignant

\footnotetext{
*Correspondence: hebosheng@126.com

${ }^{3}$ Department of Radiology, The Second Affiliated Hospital of Nantong University, No.6 Hai Er Xiang North Road, Nantong, Jiangsu 226001, P.R. China

Full list of author information is available at the end of the article
}

lymphomas. This technique not only provides the architecture of the lymph node and sufficient tissue for further examinations such as immune phenotype, molecular genetics, and molecular biology as compared with fine needle biopsy (FNB) but also saves more than $75 \%$ of costs as compared with surgical biopsy [3]. Moreover, CNB is a non-radiological and real-time examination and may be the first initial approach chosen for people who cannot endure surgery, especially for feeble or older people. Therefore, CNB has gradually been accepted as an alternative method in the diagnosis of patients with lymphoma in the United States and some Western European countries because of its good applicability, safety, and high diagnostic rate [4-11].

It has been well established that CNB is effective and useful in the diagnosis of malignant lymphomas in many organs, such as head, neck, breast, thyroid, chest, and abdomen [11-17]. However, some studies merely focused 
on isolated localization such as superficial or deep masses or merely paid close attention to one or two organs. In addition, the influence on different lengths of lesions in the diagnosis accuracy of lymphoma and its subtypes by means of CNB is still not clear.

The purpose of this study was to investigate the feasibility, accuracy, and limitations of ultrasound (US)-guided $\mathrm{CNB}$ with multiple punches in the diagnosis of lymphoma in the whole body and evaluate its clinical value. The differences between CNB and surgical group in the diagnostic accuracy rate of lymphoma and its subtypes in superficial and deep masses of the whole body were compared, as well as the influence on different lengths of lesions in the diagnosis accuracy rate of lymphoma and its subtypes in CNB group.

\section{Methods}

\section{Patients}

A total of 205 patients underwent biopsy in the Department of Ultrasound, The Cancer Hospital of Nantong University for lymphoma lesion biopsy between March 2007 and October 2013 were enrolled. All patients were randomly assigned to US-guided CNB group and surgery group. All biopsies were done on an outpatient basis, unless inpatient medical supervision is needed. In the US-guided CNB group, there were 110 patients (aged 58.4 \pm 17.3 years) consisting 60 males and 50 females. There were 62 cases of superficial masses (neck, axillary, groin, breast, vertical muscle, back) and 48 cases of deep masses (abdominal cavity, retroperitoneum). A total of 95 patients consisting 56 males and 39 females were assigned to the surgery group. The mean age was $58.9 \pm 14.4$ years. Surgery biopsies included 60 cases of superficial masses (neck, axillary, groin, breast, vertical muscle, back) and 35 cases of deep masses (abdominal cavity, retroperitoneum). However, two patients failed to be defined as subtypes of lymphoma after the first surgical biopsy. Six months later, they were confirmed as lymphoma and its subtypes in the second surgical biopsy.

Informed consents were obtained from all participants before CNB or surgery. Additionally, this study was approved by the Cancer Hospital of Nantong University medical ethics committees.

\section{Ultrasound-guided CNB}

All biopsies were conducted under the supervision of color doppler ultrasonography (Philips IU-22, Amsterdam, The Netherlands) with probe frequencies of 2 to $5 \mathrm{MHz}$ (low frequency) and 5 to $12 \mathrm{MHz}$ (high frequency). The 14-gauge (14G) and 16-gauge (16G) cutting needles and the third generation automatic biopsy gun (Bard Magnum, Covington, GA, USA) (Figure 1) were used to the biopsies. The length of needle groove was 15 or $22 \mathrm{~mm}$, depending

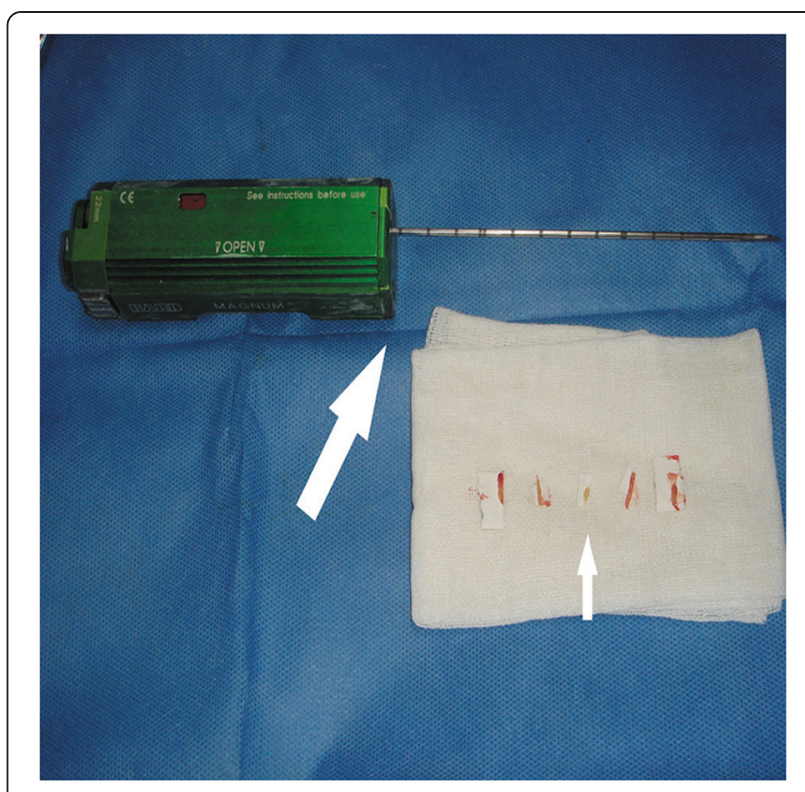

Figure 1 Bard Magnum tissue-cutting needle and the third generation automatic biopsy gun (big arrow); tissue strips obtained with CNB (small arrow).

upon the size of nodal and proximity of vessels [18]. The high-frequency probe-guided 14G core needle and lowfrequency probe-guided $16 \mathrm{G}$ core needle were used to the biopsies of superficial and deep masses, respectively (Figure 2).

Normal coagulation screening tests, including prothrombin time (PT), platelet count, and partial thromboplastin time (PTT), were performed before biopsy [19]. If the results were confirmed to be normal, CNB would be performed to the patients. The routine US was carried out to investigate the shape, echo, size, and blood supply of the masses, and the relationships among the masses, surrounding organs, and great vessels, besides the medium length of the lesions, were recorded and compared between the two groups. All the biopsies were performed by well-experienced radiologists. The local anesthesia was administered using 2\% lidocaine. Under the real-time supervision of US, the needle was inserted at the edge of the mass and the biopsy gun was instantaneously excited to collect at least three times in different parts of the mass (one needle in the center and two needles at the periphery of the mass). Intact tissue strips with a length of more than $0.5 \mathrm{~cm}$ were considered to be satisfactory samples.

Different body positions were chosen according to different sites of the masses and the locations of samples. The sampling was collected with the help of surgical knife blade, especially for the tough skin. Moreover, the sampling points should be as close as possible to the body surface and avoid the necrotic areas. The samples should be harvested from hyper-vascular tissue (Figure 3). After CNB, 


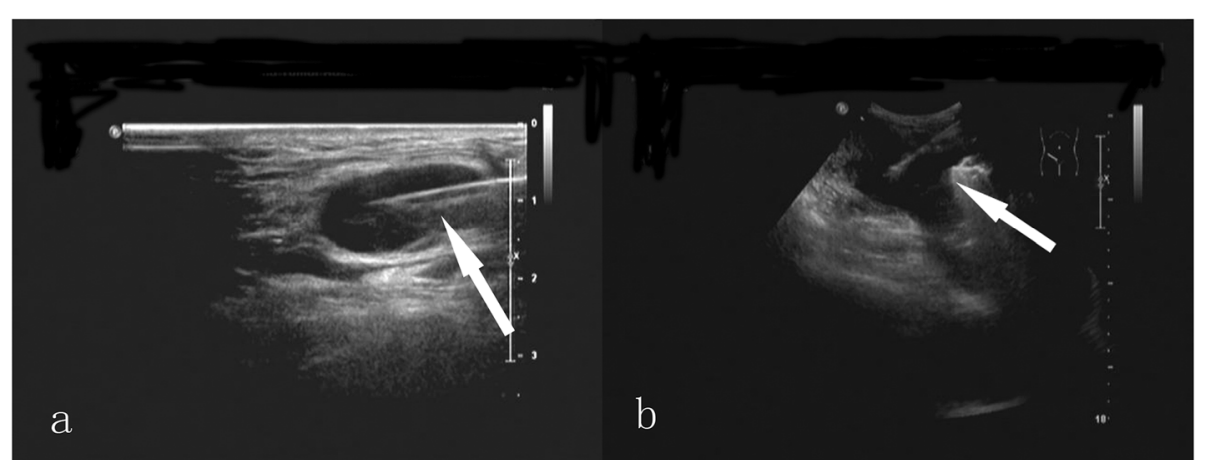

Figure 2 Ultrasonograms of the needle tracts. (a) The ultrasonogram of the needle tract in the superficial masses after ejection of biopsy gun (arrow); (b) the ultrasonogram of the needle tract in the deep masses after ejection of biopsy gun (arrow).

the puncture points should be compressed sufficiently to avoid subcutaneous hematoma.

\section{Surgical biopsy}

Adequate preoperative evaluation was done to master the indications, and normal coagulation screening tests were also assigned to the patients prior to the operation. If the results were confirmed to be normal, surgery would be allowed to perform. The surgical approach and biopsy range of masses were determined after a comprehensive analysis of clinical and imaging materials. All the surgical operation was performed by well-experienced surgeons. Excisional biopsy tissue should include the outer edge of the masses and hyper-vascular areas. The mass which was less than $3 \mathrm{~cm}$ should be wholly excised.

After the surgical biopsy, all patients in these two groups were kept under observation and then were returned to

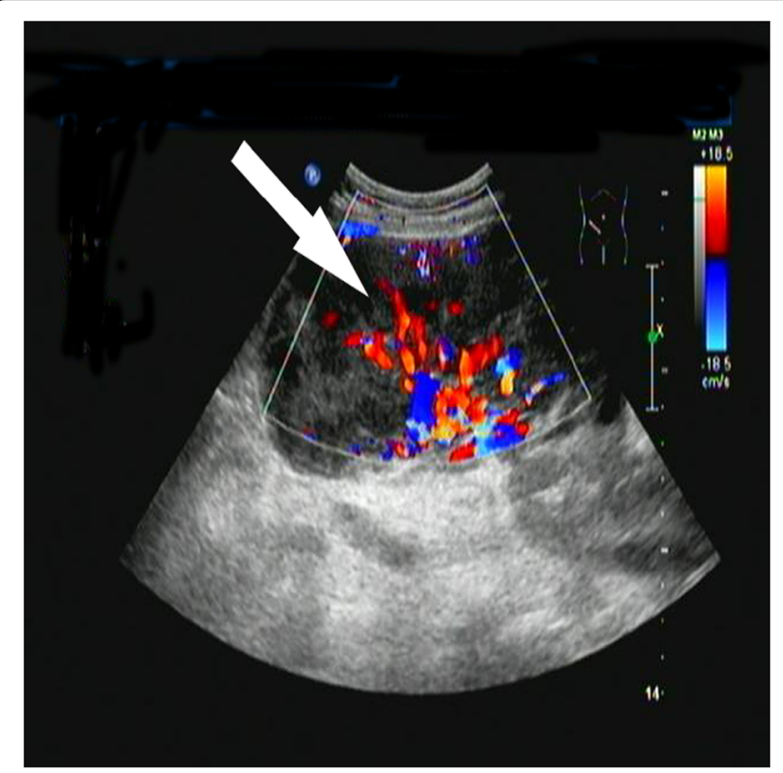

Figure $3 \mathrm{CDFI}$ shows the rich blood flow in the lesion (arrow). wards only if there was no sign or symptom of complications, such as bleeding. All patients were encouraged to call a doctor if they felt uncomfortable.

\section{Pathological diagnosis of lymphoma}

After excision of the lesions, the final diagnosis for each case was established by secondary histopathology examination which was considered as a gold standard. All samples were placed in 10\% formalin and embedded in paraffin. Serial sections $(4 \mu \mathrm{m})$ were cut and stained with hematoxylin and eosin (HE). However, histologic examination which is utilized to reveal the morphology of lymphomas is now not adequate to distinguish the different categories of lymphoma. Molecular pathology techniques, such as immunohistochemistry (IHC), have been proven effective in diagnostic approaches to malignant lymphoma. Immunophenotyping to determine lineage and clonality using more than 80 different monoclonal antibodies is now supported to distinguish the different categories of lymphoma. Hence, IHC was performed to assess the immunophenotyping in our study. The appropriate selection of the specific monoclonal antibodies should be based on morphology and relevant clinical data to avoid pitfalls in the interpretation of IHC data. In our study, IHC was implemented using EnVision two-step method on a panel of antibodies [20]: leukocyte common antigen (LCA), cluster designation (CD)20, CD79a, CD10, Bcl-6, multiple myeloma oncogene (MUM)1, Ki-67, Bcl-2, Bcl-10, CD5, CyclinD1, CD23, anaplastic lymphoma kinase (ALK)-1, CD3, CD45RO, CD56, TIA-1, and CXCL13 (Beijing Zhongshan Jinqiao Biotechnology Corporation Limited). Pathological diagnosis was referred to WHO classification of lymphatic and hematopoietic tumors.

\section{Statistical analysis}

Continuous variables were expressed as the mean \pm standard deviation (SD). Statistical analysis was performed with the STATA10.0 statistical software package (STATA Corporation, College Station, TX, USA). The accuracy rate 
was examined with Fisher's exact test. Findings with $P<$ 0.05 were considered to be statistically different.

\section{Results and discussion CNB group}

The medium length of the lesions was $7.3 \mathrm{~cm}$ (range 2.3 to $12.3 \mathrm{~cm}$ ). Majority of the lesions were greater than $3 \mathrm{~cm}(84.5 \%, 93 / 110)$ (Table 1). During the puncture, three patients presented pains and one patient appeared minor hemorrhage ( $<60 \mathrm{ml})$. There was no serious complication in all cases. The medium length of tissue strips of each case was $1.5 \mathrm{~cm}$ (range 1.2 to $1.8 \mathrm{~cm}$ ). The medium puncture number was 3.1 needles (range 2 to 6 needles) (Table 2). One patient was punctured for two needles due to local bleeding. The final pathological diagnosis included 2 cases of Hodgkin lymphoma (HL) and 108 cases of nonHodgkin lymphoma (NHL) (Table 3). Diffuse large B cell lymphoma accounted for $77.3 \%$ (85/110). Eight patients failed to be defined as subtypes of lymphoma after the first CNB (including five patients undiagnosed as lymphoma). All the eight cases were assigned to further surgical biopsy to confirm the diagnosis (one case was performed two times) (Table 4).

\section{Surgical group}

During the procedure, there were 5 patients who had hemorrhage (100 to $200 \mathrm{ml}$ ) and 10 patients presented incision pain. The final pathological diagnosis included 1 case of $\mathrm{HL}$ and 94 cases of NHL (Table 3). Two cases failed to be diagnosed as lymphoma and its subtypes in the first surgical biopsy. They were confirmed to be follicular lymphoma and diffuse large B cell lymphoma after the secondary surgical biopsy. Among the 95 cases, one patient with retroperitoneal tumor presented hypoproteinemia a week after the surgical biopsy but was improved after symptomatic treatment. The surgical incision healed slowly in one patient who had diabetes. There were no serious complications in all cases.

\section{Comparison of diagnosis accuracy rate between the two groups}

There was no statistical difference between CNB and surgical group in the diagnostic accuracy rate of lymphoma and its subtypes in superficial $(P=0.68 ; P=0.273)$ and deep masses $(P=1 ; P=0.506)$, respectively (Table 5$)$.

Table 1 Diagnostic accuracy of CNB in different lengths of lesions

\begin{tabular}{llll}
\hline Length & $\begin{array}{l}\text { Number } \\
\text { of cases }\end{array}$ & $\begin{array}{l}\text { Diagnosis accuracy } \\
\text { of lymphoma (\%) }\end{array}$ & $\begin{array}{l}\text { Diagnostic accuracy } \\
\text { of subtypes (\%) }\end{array}$ \\
\hline $\mathrm{D} \leq 3$ & 17 & $88.2(15 / 17)$ & $82.3(14 / 17)$ \\
$3<\mathrm{D} \leq 5$ & 34 & $97.1(33 / 34)$ & $94.1(32 / 34)$ \\
$\mathrm{D}>5$ & 59 & $96.6(57 / 59)$ & $95.0(56 / 59)$ \\
\hline
\end{tabular}

CNB, core needle biopsy; $D$, the length of llesions.
Table 2 Number of punctures in CNB group

\begin{tabular}{lll}
\hline Number of punctures & Number of cases & $\begin{array}{l}\text { Total number } \\
\text { of punctures }\end{array}$ \\
\hline 2 & 1 & 2 \\
3 & 98 & 294 \\
4 & 6 & 24 \\
5 & 4 & 20 \\
6 & 1 & 6 \\
Total & 110 & 346 \\
\hline
\end{tabular}

CNB, core needle biopsy.

In the CNB group, there was no statistical difference between different lengths of masses in the diagnostic accuracy rate of lymphoma and its subtypes.

\section{Discussion}

In our present study, we investigated the feasibility, accuracy, and limitations of CNB with multiple punches in the diagnosis of lymphoma in the whole body. The results suggested that US-guided CNB with no less than three punches was an accurate, safe, minimally invasive, non-radiological, fast, and cost-effective method in the

Table 3 Lymphoma subtypes in CNB group and surgical group

\begin{tabular}{|c|c|c|}
\hline Subtypes & $\begin{array}{l}\text { Number of } \\
\text { CNB group } \\
\text { cases }\end{array}$ & $\begin{array}{l}\text { Number of } \\
\text { surgical } \\
\text { group cases }\end{array}$ \\
\hline $\mathrm{NHL}$ & $108(98.2 \%)$ & $94(98.9 \%)$ \\
\hline Diffuse large B cell lymphoma & $85(78.7 \%)$ & $63(67.0 \%)$ \\
\hline Anaplastic large cell lymphoma & $7(6.5 \%)$ & $3(3.2 \%)$ \\
\hline Mantle cell lymphoma & $3(2.8 \%)$ & $8(8.5 \%)$ \\
\hline Follicular lymphoma & $2(1.9 \%)$ & $5(5.3 \%)$ \\
\hline Small B cell lymphomas & $2(1.9 \%)$ & $0(0 \%)$ \\
\hline Small lymphocytic lymphoma/leukemia & $2(1.9 \%)$ & $1(1.1 \%)$ \\
\hline Nodal marginal zone B cell lymphoma & $1(0.9 \%)$ & $1(1.1 \%)$ \\
\hline Lymphoblastic leukemia, T cell & $1(0.9 \%)$ & $0(0 \%)$ \\
\hline Unspecified peripheral T cell lymphoma & $1(0.9 \%)$ & $2(2.1 \%)$ \\
\hline Angioimmunoblastic T cell lymphoma & $1(0.9 \%)$ & $0(0 \%)$ \\
\hline Burkitt's lymphoma & $1(0.9 \%)$ & $0(0 \%)$ \\
\hline B lymphocytic lymphoma/leukemia & $1(0.9 \%)$ & $3(3.2 \%)$ \\
\hline $\begin{array}{l}\text { Plasma cell differentiation large B cell } \\
\text { lymphoma }\end{array}$ & $1(0.9 \%)$ & $0(0 \%)$ \\
\hline $\begin{array}{l}\text { B cell lymphoma of mucosa-associated } \\
\text { lymphoid tissue }\end{array}$ & $0(0 \%)$ & $5(5.3 \%)$ \\
\hline T-lymphoblastic lymphoma & $0(0 \%)$ & $2(2.1 \%)$ \\
\hline NKJT cell lymphoma & $0(0 \%)$ & $1(1.1 \%)$ \\
\hline $\mathrm{HL}$ & $2(1.8 \%)$ & $1(1.1 \%)$ \\
\hline Hodgkin lymphoma nodular sclerosing type & $2(100 \%)$ & $0(0 \%)$ \\
\hline Hodgkin lymphoma mixed cellularity type & $0(0 \%)$ & $1(100 \%)$ \\
\hline
\end{tabular}

CNB, core needle biopsy. 
Table 4 Eight cases undiagnosed as subtypes of lymphoma with CNB

\begin{tabular}{|c|c|c|c|}
\hline Location & Pathological diagnosis with CNB & Pathological diagnosis with surgical biopsy & Remarks \\
\hline Abdominal cavity & Malignant tumor, poorly differentiated carcinoma & Diffuse large B cell lymphoma & $\begin{array}{l}5 \text { years after gastric } \\
\text { cancer operation }\end{array}$ \\
\hline Abdominal cavity & Lymphoma & Diffuse large B cell lymphoma & \\
\hline Left inguinal region & Lymphadenosis & Small lymphocytic lymphoma/leukemia & Hemorrhage \\
\hline Right neck & Lymphadenosis heteromorphosis & Anaplastic large cell lymphoma & $\begin{array}{l}\text { It is confirmed after } \\
\text { the second surgery }\end{array}$ \\
\hline Left armpit & Lymphadenosis heteromorphosis & Small B cell lymphomas & \\
\hline Right armpit & Chronic inflammation accompanied with lymphadenosis & Angioimmunoblastic T cell lymphoma & \\
\hline Left armpit & Lymphoma & Unspecified peripheral T cell lymphoma & \\
\hline Right neck & Lymphoma & Nodal marginal zone B cell lymphoma & Rare type \\
\hline
\end{tabular}

CNB, core needle biopsy.

evaluation of lymphoma and its subtypes compared with surgical approach.

US-guided CNB is a non-radiological and real-time examination, which can provide blood flow information of lesions ensuring the accuracy of sampling. Moreover, the obtained sample is intact, and the cells are not compressed which is helpful for pathological examination [21]. In addition, CNB enables us to streamline patient care and reduce operating time and expense compared with surgery [21]. Previous study indicated that the success rate of $\mathrm{CNB}$ was significantly higher than FNB (37.6\%) [22] and CT-guided CNB (71.5\%) [23]. A large number of studies have documented that CNB is an effective and reliable procedure with a high diagnostic yield for lymphoma $[9,24,25]$.In our studies, we evaluated the diagnostic accuracy of lymphoma and its subtypes in superficial and deep masses using CNB and surgery. The results showed that there was no statistical difference $(P=0.273$, and $P=0.506$, respectively), indicating that $\mathrm{CNB}$ was not only suited for deep masses but also for superficial masses. In the past, surgery was advocated for superficial masses; however, our study provided some evidence to back up CNB for diagnosing superficial masses. What is more, we also found that there was no significant difference between different lengths of

Table 5 Diagnostic accuracy of lymphoma and its subtypes between CNB and surgical group in superficial and deep masses

\begin{tabular}{lllll}
\hline Location & Diagnosis & CNB group & Surgical group & $\boldsymbol{P}$ \\
\hline Superficial masses & Lymphoma & $93.5(58 / 62)$ & $96.6(58 / 60)$ & 0.68 \\
\cline { 2 - 5 } Deep masses & Subtypes & $90.3(56 / 62)$ & $96.6(58 / 60)$ & 0.273 \\
& Lymphoma & $97.9(47 / 48)$ & $100(35 / 35)$ & 1 \\
\multirow{2}{*}{ Total } & Subtypes & $95.8(46 / 48)$ & $100(35 / 35)$ & 0.506 \\
& Lymphoma & $95.4(105 / 110)$ & $97.9(96 / 98)$ & 0.454 \\
& Subtypes & $92.7(102 / 110)$ & $97.9(96 / 98)$ & 0.11 \\
\hline
\end{tabular}

CNB, core needle biopsy. lesions in the diagnostic accuracy rate of lymphoma and its subtypes.

Hemorrhage is the most common complication of needle biopsy, and the incidence is $0.8 \%$ to $3.0 \%$ [26,27]. In our study, there was only one patient who had minor hemorrhage $(<60 \mathrm{ml})$ and three patients presented pains. In addition, there were no serious complications in all cases. Whereas, there were 5 patients who had hemorrhage (100 to $200 \mathrm{ml}$ ) and 10 patients presented incision pain in the surgery group. On the whole, the US-guided CNB reduced the complications as compared with surgical biopsy.

Although percutaneous US-guided CNB is currently the best way to obtain positive histopathological diagnosis of lymphoma under the non-surgical conditions, several points should be noted. First of all, CNB should be performed by well-experienced radiologists to acquire adequate sample. Secondly, sufficient and high-quality sample volume may be the key factors affecting the accuracy rate of CNB $[28,29]$. Therefore, sampling sites should be chosen in or closed to the rich blood supply areas and in the periphery of the lesion. Thirdly, USguided CNB should be used for the lesions visualized clearly by ultrasound; however, for the lesions which could not be displayed clearly by US or negative results of $\mathrm{CNB}$, local surgical biopsy should be performed. Fourthly, the application of $14 \mathrm{G}$ and $16 \mathrm{G}$ needles and automatic biopsy gun also should be noteworthy. In our study, 14G and 16G were used to the biopsies of superficial and deep masses, respectively. The choice of biopsy needle size mainly depends on the size and localization of lesions [14]. Generally speaking, large core needles (usually 14G) are utilized to superficial biopsies, whereas smaller needles (18 or 16G) are performed on deep biopsies [30]. The last point is multi-point sampling. Usually, lymphoma is characterized by multifocal in origin; therefore, multi-point sampling should be conducted to acquire effective and adequate tissue. Bolivar et al. [31] suggested that each lesion should be punctured for no less than three needles in the diagnostic value of US- 
guided 14G CNB in non-palpable suspicious breast lesions. In line with their study, we also found that no less than three punches may be effective for CNB in diagnosing lymphoma in our study.

In the CNB group, eight patients failed to be defined as subtypes of lymphoma after the first CNB (including five patients undiagnosed as lymphoma). The eight patients then underwent surgical biopsy to confirm the final diagnosis and subtypes of lymphoma. Of the five patients undiagnosed as lymphoma, one patient was confirmed finally to have anaplastic large cell lymphoma. The reasons for the failure of diagnosis with CNB may be relevant to reactive hyperplasia in the sampling areas or the sample was not a representative of the characteristics of lymphoma. One patient had minor hemorrhage and was punctured with only two needles resulting in the reduction of sample volume. For the other one case, the tumor was the largest among all patients enrolled in this study and the sample was probably punctured in necrotic tissues. In the three cases failed to be confirmed to be subtypes of lymphoma, one case was a rare type (nodal marginal zone B cell lymphoma) and the diagnosis may be difficult, and the other two cases may be relevant to less sample volume.

However, there were several limitations in our study. The lesions were only divided into superficial and deep masses; the specific location of the enlarged lymph nodes was not taken into consideration. The lesions which were not displayed clearly by US were not enrolled in the CNB group. All of these may lead to biased results. In addition, our study did not intensively investigate the quality control factors of the golden standard (pathological diagnosis).

\section{Conclusions}

In summary, US-guided CNB with no less than three punches is an accurate, safe, minimally invasive, convenient, fast, and low-cost method in the evaluation of lymphoma and its subtypes. It should be used as the acceptable alternative to surgical biopsy to obtain histopathological samples in the patients with suspected lymphoma.

\section{Abbreviations}

CNB: core needle biopsy; FNB: fine needle biopsy; HE: hematoxylin and eosin; HL: Hodgkin lymphoma; IHC: immunohistochemistry; NHL: non-Hodgkin Iymphoma; PT: prothrombintime; PTT: partial thromboplastin time; SD: standard deviation..

\section{Competing interests}

The authors declare that they have no competing interests.

\section{Authors' contributions}

$\mathrm{YH}$ and XX participated in the design of this study and performed the statistical analysis. $\mathrm{YH}, \mathrm{BH}$ and $\mathrm{YX}$ carried out the study, together with $\mathrm{XJ}$, collected important background information, and drafted the manuscript. XC and QZ conceived this study, participated in the design, and helped to draft the manuscript. All authors read and approved the final manuscript.

\section{Acknowledgements}

This work was supported by The Society and Development Planning of Nantong (Fund number: S11916).

\section{Author details}

'Department of Ultrasound, The Cancer Hospital of Nantong University, No. 30 Tongyang North Road, Pingchao Town, Tongzhou District, Nantong 226361, China. ${ }^{2}$ Department of Ultrasound, The First Affiliated Hospital of Nantong University, No. 20 Xisi Road, Nantong 226001, China. ${ }^{3}$ Department of Radiology, The Second Affiliated Hospital of Nantong University, No.6 Hai Er Xiang North Road, Nantong, Jiangsu 226001, P.R. China. ${ }^{4}$ Department of Oncology, The Cancer Hospital of Nantong University, No. 30 Tongyang North Road, Pingchao Town, Tongzhou District, Nantong 226361, China. ${ }^{5}$ Department of Pathology, The Cancer Hospital of Nantong University, No. 30 Tongyang North Road, Pingchao Town, Tongzhou District, Nantong 226361, China.

Received: 19 December 2014 Accepted: 7 March 2015

Published online: 27 March 2015

\section{References}

1. Halliday T, Baxter G. Lymphoma: pictorial review. I. Eur Radiol. 2003;13:1154-64.

2. Skoog L, Tani E. Diagnosis of lymphoma by fine-needle aspiration cytology using the revised European-American classification of lymphoid neoplasms. Cancer. 2000;90:320-3.

3. Lachar WA, Shahab I, Saad AJ. Accuracy and cost-effectiveness of core needle biopsy in the evaluation of suspected lymphoma: a study of 101 cases. Arch Pathol Lab Med. 2007;131:1033-9.

4. de Kerviler E, de Bazelaire C, Mounier N, Mathieu O, Brethon B, Briere J, et al. Image-guided core-needle biopsy of peripheral lymph nodes allows the diagnosis of lymphomas. Eur Radiol. 2007;17:843-9.

5. Demharter J, Neukirchen S, Wagner T, Schlimok G, Bohndorf K, Kirchhof K. Do ultrasound-guided core needle biopsies of lymph nodes allow for subclassification of malignant lymphomas? Rofo. 2007;179:396-400.

6. Li L, Wu QL, Liu LZ, Mo YX, Xie CM, Zheng L, et al. Value of CT-guided core-needle biopsy in diagnosis and classification of malignant lymphomas using automated biopsy gun. World J Gastroenterol. 2005;11:4843-7.

7. Kim BM, Kim EK, Kim MJ, Yang WI, Park CS, Park SI. Sonographically guided core needle biopsy of cervical lymphadenopathy in patients without known malignancy. J Ultrasound Med. 2007;26:585-91.

8. Zinzani PL, Corneli G, Cancellieri A, Magagnoli M, Lacava N, Gherlinzoni F, et al. Core needle biopsy is effective in the initial diagnosis of mediastinal lymphoma. Haematologica. 1999;84:600-3.

9. Ben-Yehuda D, Polliack A, Okon E, Sherman Y, Fields S, Lebenshart P, et al. Image-guided core-needle biopsy in malignant lymphoma: experience with 100 patients that suggests the technique is reliable. J Clin Oncol. 1996;14:2431-4

10. Pappa VI, Hussain HK, Reznek RH, Whelan J, Norton AJ, Wilson AM, et al. Role of image-guided core-needle biopsy in the management of patients with lymphoma. J Clin Oncol. 1996;14:2427-30.

11. de Larrinoa AF, del Cura J, Zabala R, Fuertes E, Bilbao F, Lopez Jl. Value of ultrasound-guided core biopsy in the diagnosis of malignant lymphoma. J Clin Ultrasound. 2007;35:295-301.

12. Walsh S, Lowery AJ, Evoy D, McDermott EW, Prichard RS. Thyroid lymphoma: recent advances in diagnosis and optimal management strategies. Oncologist. 2013;18:994-1003.

13. Stewart CJ, Coldewey J, Stewart IS. Comparison of fine needle aspiration cytology and needle core biopsy in the diagnosis of radiologically detected abdominal lesions. J Clin Pathol. 2002;55:93-7.

14. Screaton NJ, Berman LH, Grant JW. Head and neck lymphadenopathy: evaluation with US-guided cutting-needle biopsy. Radiology. 2002;224:75-81.

15. Janes SE, Lengyel JA, Singh S, Aluwihare N, Isgar B. Needle core biopsy for the assessment of unilateral breast masses in men. Breast. 2006;15:273-5.

16. Zinzani PL, Colecchia A, Festi D, Magagnoli M, Larocca A, Ascani S, et al. Ultrasound-guided core-needle biopsy is effective in the initial diagnosis of lymphoma patients. Haematologica. 1998;83:989-92.

17. Huang PC, Liu CY, Chuang WY, Shih LY, Wan YL. Ultrasound-guided core needle biopsy of cervical lymphadenopathy in patients with lymphoma: the clinical efficacy and factors associated with unsuccessful diagnosis. Ultrasound Med Biol. 2010;36:1431-6. 
18. Britton PD, Provenzano E, Barter S, Gaskarth M, Goud A, Moyle P, et al. Ultrasound guided percutaneous axillary lymph node core biopsy: how often is the sentinel lymph node being biopsied? Breast. 2009;18:13-6.

19. Sklair-Levy M, Amir G, Spectre G, Lebensart P, Applbaum Y, Agid R, et al. Image-guided cutting-edge-needle biopsy of peripheral lymph nodes and superficial masses for the diagnosis of lymphoma. J Comput Assist Tomogr. 2005;29:369-72.

20. Sabattini E, Bisgaard K, Ascani S, Poggi S, Piccioli M, Ceccarelli C, et al. The EnVision++ system: a new immunohistochemical method for diagnostics and research. Critical comparison with the APAAP, ChemMate, CSA, LABC, and SABC techniques. J Clin Pathol. 1998;51:506-11.

21. Ganott MA, Zuley ML, Abrams GS, Lu AH, Kelly AE, Sumkin JH, et al. Ultrasound guided core biopsy versus fine needle aspiration for evaluation of axillary lymphadenopathy in patients with breast cancer. ISRN Oncol. 2014:2014:703160

22. Hehn ST, Grogan TM, Miller TP. Utility of fine-needle aspiration as a diagnostic technique in lymphoma. J Clin Oncol. 2004:22:3046-52.

23. Sklair-Levy M, Polliack A, Shaham D, Applbaum YH, Gillis S, Ben-Yehuda D, et al. CT-guided core-needle biopsy in the diagnosis of mediastinal lymphoma. Eur Radiol. 2000;10:714-8.

24. de Kerviler E, Guermazi A, Zagdanski AM, Meignin V, Gossot D,

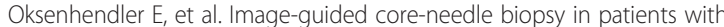
suspected or recurrent lymphomas. Cancer. 2000;89:647-52.

25. Demharter J, Muller P, Wagner T, Schlimok G, Haude K, Bohndorf K. Percutaneous core-needle biopsy of enlarged lymph nodes in the diagnosis and subclassification of malignant lymphomas. Eur Radiol. 2001;11:276-83.

26. Youk JH, Kim EK, Kwak JY, Son EJ. Atypical papilloma diagnosed by sonographically guided 14-gauge core needle biopsy of breast mass. AJR Am J Roentgenol. 2010;194:1397-402.

27. Bahn YE, Lee SK, Kwon SY, Kim SP. Sonographic appearances of mucosa-associated lymphoid tissue lymphoma of the submandibular gland confirmed with sonographically guided core needle biopsy. J Clin Ultrasound. 2011:39:228-32.

28. Schueller G, Jaromi S, Ponhold L, Fuchsjaeger M, Memarsadeghi M, Rudas $M$, et al. US-guided 14-gauge core-needle breast biopsy: results of a validation study in 1352 cases. Radiology. 2008;248:406-13.

29. Abe H, Schmidt RA, Kulkarni K, Sennett CA, Mueller JS, Newstead GM. Axillary lymph nodes suspicious for breast cancer metastasis: sampling with US-guided 14-gauge core-needle biopsy-clinical experience in 100 patients. Radiology. 2009;250:41-9.

30. de Kerviler E, Benet C, Briere J, de Bazelaire C. Image-guided needle biopsy for diagnosis and molecular biology in lymphomas. Best Pract Res Clin Haematol. 2012;25:29-39.

31. Bolivar AV, Alonso-Bartolome P, Garcia EO, Ayensa FG. Ultrasound-guided core needle biopsy of non-palpable breast lesions: a prospective analysis in 204 cases. Acta Radiol. 2005:46:690-5.

\section{Submit your next manuscript to BioMed Central and take full advantage of:}

- Convenient online submission

- Thorough peer review

- No space constraints or color figure charges

- Immediate publication on acceptance

- Inclusion in PubMed, CAS, Scopus and Google Scholar

- Research which is freely available for redistribution 